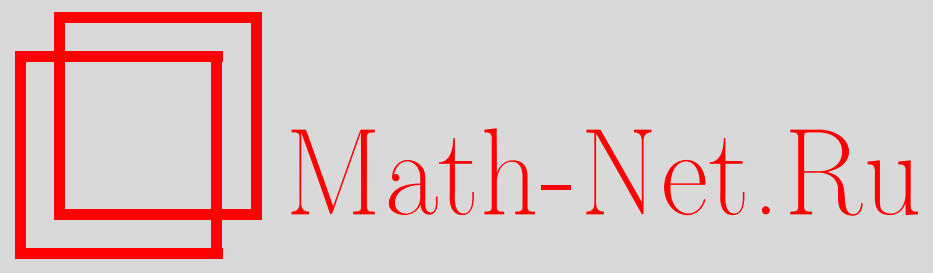

С. Ю. Доброхотов, А. И. Шафаревич, Туннельное расщепление спектра операторов ЛапласаБельтрами на двумерных поверхностях с квадратично интегрируемым геодезическим потоком, Функи. анализ и его прил., 2000, том 34, выпуск 2, 67-69

DOI: https://doi.org/10.4213/faa297

Использование Общероссийского математического портала MathNet.Ru подразумевает, что вы прочитали и согласны с пользовательским соглашением

http://www . mathnet.ru/rus/agreement

Параметры загрузки:

IP : 54.198 .55 .26

26 апреля 2023 г., 14:19:59

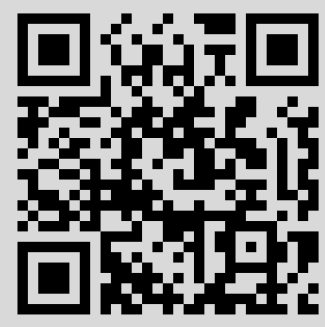


1984. 3. Арнольд В. И., Васильев В. А., Горюнов В. В., Ляшко О. В. Особенности I. Локальная и глобальная теория. Динамические системы VI. Итоги науки и техники. Соврем. пробл. матем. Фундам. напр., т. 6, ВИНИТИ, М., 1988. 4. Арнольд В. И., Васильев В. А., Горюнов В. В., Ляшко О. В. Особенности II. Классификация и приложения. Динамические системы VIII. Итоги науки и техники. Соврем. пробл. матем. Фундам. напр., т. 39, ВИНИТИ, М., 1989. 5. Bruce J. W. Bull. London Math. Soc., 17, No. 5, 257-262 (1985). 6. Damon J. N. Mem. Amer. Math. Soc., 50, No. 306, 1-88 (1984). 7. Горюнов B. В. Функц. анализ и его прил., 15, вып. 2, 1-8 (1981). 8. Frühbis-Krueger A. Preprint, Universität Kaiserslautern, 1998. 9. Looijenga E. J. N. Invent. Math., 23, 105-116 (1974). 10. Закалюкин B. M. Функц. анализ и его прил., 10, вып. 2, 69-70 (1976).

Department of Mathematical Sciences

Division of Pure Mathematics

Поступило в редакцию

The University of Liverpool

8 сентября 1998 г.

e-mail: goryunov@liv.ac.uk

УДК 517.98

\title{
Туннельное расщепление спектра операторов Лапласа-Бельтрами на двумерных поверхностях с квадратично интегрируемым геодезическим потоком
}

\author{
(C) 2000. С. Ю. Доврохотов, А. И. ШАФАРЕВИЧ
}

Пусть $M$ - тор или сфера с квадратично интегрируемым по Лиувиллю геодезическим потоком, т. е. в кокасательном расслоении $T^{*} M$ существует независимый с гамильтонианом $H=|p|^{2}$ квадратичный по импульсам $p$ интеграл $F$ (см. [1-3]). Метрика на $M$ приводится к лиувиллеву виду $d s^{2}=$ $\left(u_{1}\left(x_{1}\right)-u_{2}\left(x_{2}\right)\right)\left(d x_{1}^{2}+d x_{2}^{2}\right), u_{1}-u_{2}>0$, причем координаты $\left(x_{1}, x_{2}\right)$ определены на торе $\widetilde{M}$, конечнолистно накрывающем исходную поверхность $M$. Пусть $\widetilde{F}, \widetilde{H}$ — поднятия интегралов $F, H$ на $T^{*} \widetilde{M}$. Множество уровня $\{\widetilde{H}=E, \widetilde{F}=\mathscr{E}\}$, где $\min u_{1}<\mathscr{E} / E<\max u_{1}$, состоит из двух торов $\widetilde{\Lambda}^{ \pm}$, накрывающих лиувиллевы торы геодезического потока. Инволюция $p \rightarrow-p$ переводит в себя лиувиллево слоение, причем отдельные лиувиллевы торы, вообще говоря, переставляются между собой.

Пусть $\Delta$ - оператор Лапласа-Бельтрами на $M$; каждый неособый лиувиллев тор $\Lambda$ геодезического потока, удовлетворяющий условию квантования [5], определяет в квазиклассическом пределе $h \rightarrow 0$ квазимоду $\psi \in L^{2}(M), E \in \mathbb{R}$ оператора $-h^{2} \Delta$. При этом асимптотическая собственная функция $\psi$ имеет вид $\psi=K_{\Lambda}^{a}(1)$, где $K_{\Lambda}^{a}$ - канонический оператор Маслова [5] на лагранжевом торе $\Lambda$ с произвольной отмеченной точкой $a$; асимптотическое собственное число $E$ вычисляется из условий квантования (явные формулы для случая $M=T^{2}$ см., например, в [6]). Рассмотрим пару различных торов $\Lambda^{ \pm}$, переходящих друг в друга под действием инволюции $p \rightarrow-p$. Они одновременно удовлетворяют (или не удовлетворяют) условиям квантования, причем числа $E$, определяемые этими торами, совпадают. Переменные в спектральной задаче для оператора Лапласа разделяются; используя результаты [4], нетрудно доказать существо- 
вание двух собственных чисел $\lambda^{ \pm}=E+O\left(h^{2}\right)$ оператора $-h^{2} \Delta$, расстояние между которыми есть $O\left(h^{\infty}\right)$, а также получить асимптотическую формулу для этого расстояния и для собственных функций, соответствующих $\lambda^{ \pm}$. Наше наблюдение состоит в том, что разность $\lambda^{+}-\lambda^{-}$выражается через характеристики комплексных лагранжевых многообразий.

Для упрощения формулировки результатов будем считать, что $u_{i}$ - тригонометрические полиномы. Комплексифицируем тор $\widetilde{M}$, накрывающий исходную поверхность $M$, полагая ${ }^{\mathbb{M}}=\mathbb{C}^{2} /(2 \pi \mathbb{Z} \oplus 2 \pi \mathbb{Z})$, и рассмотрим комплексное фазовое пространство $\Phi={ }^{\mathbb{C}} T^{*} \widetilde{M}=\mathbb{C} \widetilde{M} \times \mathbb{C}^{2}$. Уравнения $\widetilde{H}=E, \widetilde{F}=\mathscr{E}$ задают в $\Phi$ комплексное лагранжево многообразие $L$. Расщепление собственных чисел оператора Лапласа выражается через следующие объекты на этом многообразии: 1) голоморфную 1-форму $\theta=(w, d z)$, где $z$ - комплексные координаты на ${ }^{\mathbb{M}} \widetilde{M}$, а $w_{j}$ - комплексные импульсы; 2) два голоморфных векторных поля $v_{1}, v_{2}, v_{j}$ определено в $\Phi$ как гамильтоново поле с гамильтонианом $\left.w_{j}^{2}+(-1)^{j}\left(\mathscr{E}-E u_{j}\left(z_{j}\right)\right) ; 3\right)$ келерову метрику $\langle\cdot, \cdot\rangle$, в которой поля $v_{1}, v_{2}$ образуют ортонормированный базис; 4) векторное поле $\xi$, определенное по форме $\theta$ и метрике $\langle\cdot, \cdot\rangle, \theta(\zeta)=i\langle\zeta, \xi\rangle$ для любого касательного вектора $\zeta$. Келерова метрика определяет на $L$ риманову и симплектическую структуры; относительно этих структур поле $\xi$ является одновременно локально гамильтоновым и локально градиентным - его локальный гамильтониан есть $\int \operatorname{Re} \theta$, а локальный потенциал равен $\int \operatorname{Im} \theta$.

Пусть $b$ - особая точка поля $\xi$; рассмотрим отрезок траектории этого поля, соединяющий $b$ с некоторой точкой на вещественном торе $\widetilde{\Lambda}^{+}$. Действуя на этот отрезок инволюцией $w \rightarrow-w$ и комплексным сопряжением, получим одномерный цикл на многообразии $L$. Проинтегрируем по каждому такому циклу форму $\operatorname{Im} \theta$ и обозначим через $\Gamma$ цикл, для которого этот интеграл минимален. Назовем его туннельньлм ииклом на многообразии $L$, точки $a^{ \pm}$его пересечения с торами $\widetilde{\Lambda}^{ \pm}-$стоксовыми точками на торах $\widetilde{\Lambda}^{ \pm}$(они определены однозначно для каждого туннельного цикла), а число $\rho=\frac{1}{2} \int \operatorname{Im} \theta-$ туннельным расстоянием между $\Lambda^{+}$и $\Lambda^{-}$.

В формулу для расщепления собственных чисел, помимо туннельного расстояния, входит еще значение некоторого коцикла $\varkappa \in H^{1}\left(\widetilde{\Lambda}^{+}, \mathbb{R}\right)$ на некотором цикле $\eta_{0}$. Они определяются следующим образом. Пусть $\gamma_{1}, \gamma_{2}$ - базис циклов на торе $\widetilde{\Lambda}^{+}$; продолжим его гладким образом на соседние торы лиувиллева слоения и рассмотрим соответствующий вектор частот $\omega$ гамильтонова поля, задающего геодезический поток. Положим $\varkappa(\eta)=(\omega, m)=\omega_{1} m_{1}+\omega_{2} m_{2}$ для любого $\eta \in H_{1}\left(\widetilde{\Lambda}^{+}, \mathbb{Z}\right)$, где $m_{j}$ - индексы пересечений $\eta$ с базисными циклами $\gamma_{1}, \gamma_{2}$ (коцикл $\varkappa$ не зависит от выбора базиса $\gamma$ ). Наконец, определим цикл $\eta_{0} \in H_{1}\left(\widetilde{\Lambda}^{+}, \mathbb{Z}\right)$. На каждой комплексной траектории $v_{j}$ построим свое градиентно-гамильтоново поле $\xi_{j}$, ограничивая форму $\theta$ и келерову метрику $\langle\cdot, \cdot\rangle$ на эту двумерную поверхность. Рассмотрим отрезки сепаратрис поля $\xi_{j}$, соединяющие множество $\Sigma \subset L$ особых точек естественной проекции $L \rightarrow \mathbb{C}^{M}$ с тором $\widetilde{\Lambda}^{+}$и такие, что интеграл по каждому такому отрезку от $\operatorname{Im} \theta$ равен $\rho / 2$. Их точки пересечения с $\widetilde{\Lambda}^{+}$образуют цикл, который мы и обозначим через $\eta_{0}$. 
ТеОРема. Пусть туннельный иикл Г единствен с точностью до гомотопии. Пусть, кроме того, на торах $\Lambda^{ \pm}$выполнены условия квантования. Тогда оператор $-h^{2} \Delta$ на $M$ имеет два собственньх числа $\lambda^{ \pm}=E+O\left(h^{2}\right)$, причем расстояние между ними имеет вид

$$
\lambda^{+}-\lambda^{-}=\frac{h}{\pi} e^{-\rho / h}\left(\varkappa\left(\eta_{0}\right)+O(h)\right) .
$$

Собственные функции $\varphi^{ \pm}$, отвечающие этим собственным числам, имеют вид $\varphi^{ \pm}=\left(K_{\Lambda^{+}}^{a^{+}}(1) \pm K_{\Lambda^{-}}^{a^{-}}(1)\right)+O(h)$, где $K-$ канонический оператор Маслова, а $a^{ \pm}$- проекиии на $\Lambda^{ \pm}$стоксовых точек, отвечаюших одному и тому же туннельному ииклу.

ЗАмечАниЕ. Сепаратрисы градиентно-гамильтонова поля $\xi$ играют ту же роль в туннельных эффектах, что и антистоксовы канонические пути в одномерном случае [4]; отметим, что сепаратрисы векторного поля $i \xi$ (также градиентно-гамильтонова) аналогичны линиям Стокса.

\title{
ЛИТЕРАТУРА
}

1. Козлов В. В. Симметрии, топология и резонансы в гамильтоновой механике. Изд-во Удмуртского гос. унив., 1995. 2. Колокольчов В. Н. Изв. АН СССР, сер. матем., 46, № 5, 994-1010 (1982). 3. Бабенко И. К., Нехорошев Н. Н. Матем. заметки, 58, № 2, 643-652 (1995). 4. Федорюк M. В. Асимптотические методы для линейных обыкновенных дифференциальных уравнений. Наука, М., 1983. 5. Маслов В. П., Федорюк $M$. . В. Квазиклассическое приближение для уравнений квантовой механики. Наука, М., 1976. 6. Косыгин Д. В., Минасов А. А., Синай Я. Г. УМН, 48, вып. 4, 3-130 (1993).

Институт проблем механики РАН

Московский государственный университет,

Поступило в редакцию механико-математический факультет

24 июня 1998 г.

\section{УДК 514.7}

\section{Лоренцевы мировые линии и производная Шварца}

\author{
(c) 2000. К. ДювАль, В. Ю. ОвСИЕнко
}

Цель настоящей заметки - установить связь между классической производной Шварца и геометрией лоренцевых поверхностей постоянной кривизны.

1. Отправной точкой для нас послужил следующий факт (установленный совместно с Л. Гийо). Рассмотрим кривую, заданную уравнением $y=f(x)$, на плоскости с лоренцевой метрикой $\mathrm{g}=d x d y$. Если $f^{\prime}(x)>0$, то ее лоренцева кривизна, имеющая вид $\varrho(x)=f^{\prime \prime}(x)\left(f^{\prime}(x)\right)^{-3 / 2}$, обладает следующим замечательным свойством:

$$
\sqrt{f^{\prime}(x)} \varrho^{\prime}(x)=S(f)(x)
$$

где

$$
S(f)(x)=\frac{f^{\prime \prime \prime}(x)}{f^{\prime}(x)}-\frac{3}{2}\left(\frac{f^{\prime \prime}(x)}{f^{\prime}(x)}\right)^{2}
$$

